\title{
Contextos de interacción educativa y desarrollo de niños preescolares
}

\section{Educational Interaction and Development Contexts in Pre-school Children}

\author{
Hernán Sánchez Ríos \\ Universidad del Valle, Colombia. \\ (Rec: octubre de 2019 - Acept: mayo de 2020)
}

\section{Resumen}

Este artículo establece relaciones entre el desarrollo cognitivo de 405 niños ${ }^{1}$ de 3, 4 y 5 años y sus contextos educativos en la modalidad Hogares Comunitarios del Instituto Colombiano de Bienestar Familiar (HC-ICBF). Desde el enfoque sistémico de U. Bronfenbrenner se establecen relaciones entre indicadores de desarrollo de los niños y los descriptores de los contextos educativos denominados HC - ICBF. Los indicadores se establecen a partir de niveles diferenciados de estrategias de inferencia en la resolución de un problema, anclado a prácticas culturales específicas. Estadísticas no paramétricas y análisis de correspondencia múltiples (ACM) permitieron establecer que el desarrollo cognitivo de los niños preescolares tiene relaciones significativas con las expectativas que el cuidador tiene respecto de la educación de los niños. Estos datos dialogan con perspectivas de la psicología del desarrollo que vinculan los procesos de construcción de conocimiento a contextos culturales específicos.

Palabras claves: Desarrollo cognitivo, contextos de interacción, procesos inferenciales.

\begin{abstract}
This article links the cognitive development of 405 children between 3 and 5 years old and their educational contexts under the modality of Community Homes of the Colombian Institute of Family Wellbeing (HC-ICBF). The systemic approach of Bronfenbrenner University establishes relations between development indicators of children and the descriptors of educational contexts called HC-ICBF. Indicators are established based on differentiated levels of inference strategies for problem resolutions concerning specific cultural practices. Non-parametric statistics and multiple correspondence analysis helped to determine that cognitive development of pre-school children have significant relationships with the expectation the caregiver has about children's education. These data relate to the approaches to developmental psychology that associate the knowledge construction processes with specific cultural contexts.
\end{abstract}

Keywords: Cognitive development, interaction contexts, inference processes.

Correspondencia a: Hernán Sánchez, hernan.sanchez@correounivalle.edu.co 


\section{Introducción}

Este artículo presenta datos de un estudio ${ }^{2}$ que establece relaciones entre el desarrollo cognitivo de 405 niños de 3, 4 y 5 años que viven en dos ciudades colombianas y sus contextos educativos de interacción en la modalidad Hogares Comunitarios del Instituto Colombiano de Bienestar Familiar (HCICBF).

El balance de la investigación en este campo de conocimiento permite identificar tendencias diferenciadas en los estudios de desarrollo cognitivo en contextos culturales específicos. La primera tendencia reporta un número significativo de investigaciones que proporcionan evidencia empírica acerca de la incidencia que tienen los contextos de interacción educativa en el desarrollo de los procesos psicológicos superiores de niños preescolares (Gauvain \& Rogoff, 1989; Rogoff, 1993; Gauvain, 1995; Gauvain, 2001; Gauvain \& Perez, 2005). En esta modalidad de estudios se examina la relevancia que tienen compañeros más experimentados en la resolución conjunta de problemas escolares. Entonces, se revela la contribución del otro en el uso de herramientas culturales y en el desarrollo del pensamiento (Saxe, Guberman \& Gwarhart 1987; Gauvain, 2001; Gleason \& Schauble, 2000). Algunas investigaciones indagan la incidencia que tiene en el desarrollo psicológico de los niños la regulación de su comportamiento o las nuevas interacciones que surgen a partir del ingreso al sistema educativo (Hojholt, 2005; Lippard, Riley \& Hughes-Belding, 2016).

Otros estudios reconstruyen, en trayectorias escolares, la incidencia de la calidad del cuidado infantil en el desarrollo cognitivo y social de los niños (Burchinal, et al, 2001; Greenfield \& Maynarda, 2003; Carrol, Fuller, Loeb \& Kagan, 2004; Gauvain \& Perez, 2005; Calero et al. 2013). Así, el estudio longitudinal de Burchinal, et al. (2001) estableció la incidencia de la calidad de la atención de Centros Comunitarios de Cuidado Infantil en los patrones de desarrollo cognitivo y socioemocional de 733 niños, entre 4 y 8 años, durante el jardín de infantes y, en algunos casos, hasta el segundo grado de la básica primaria. En esta línea de trabajo, algunas investigaciones establecieron los efectos del nivel socio económico de las familias, la ocupación de los padres y la escolaridad temprana en el rendimiento escolar y en el aprendizaje de los niños (Caspary, Fuller, Gauthier \& Kagan, 2002; Carrol, Fuller, Loeb \& Kagan, 2003; Carrol, Fuller, Loeb \& Kagan, 2004).

En esta discusión, tiene una contribución relevante el modelo ecológico de desarrollo humano de Urie Bronfenbrenner. Esta teoría permite establecer la incidencia que tienen los ambientes ecológicos en el cambio y la continuidad de los procesos de desarrollo individual.

En su modelo ecológico, Bronfenbrenner (1987) distingue cuatro sistemas que se relacionan entre sí como una organización en círculos concéntricos que inciden en el desarrollo humano. En primera instancia, describe los microsistemas como patrones de actividades, roles y relaciones interpersonales en los que la persona participa en un entorno determinado. Las interrelaciones entre dos o más entornos en los que las personas interactúan constituyen el mesosistema; ilustra esta dimensión, las relaciones entre actores, prácticas, roles y modali- dades de interacción entre la familia y el Centro de Cuidado y Protección de los niños. Los siguientes sistemas ecológicos no afectan directamente al individuo, sino a sus entornos. Así, el exosistema se refiere a uno o más entornos que no incluyen a la persona en desarrollo como participante activo, pero en los cuales ocurren hechos que afectan su entorno directo. De esta manera, son ejemplos de este sistema: las jornadas laborales de los padres o los programas de protección y cuidado que tienen los padres para dejar a sus hijos mientras trabajan. Por último, el macrosistema está constituido por el sistema de creencias o ideologías que sustentan los anteriores.

Los contextos culturales de interacción en los cuales los niños menores de siete años construyen conocimiento pueden ser: la familia, los centros de cuidado y protección, los preescolares, los hogares comunitarios, la escuela y la comunidad. Estos microsistemas ofrecen condiciones contextuales que pueden o no potenciar el desarrollo de los niños. Desde la perspectiva ecológica del desarrollo, los microsistemas se definen en función del marco de actividades molares, es decir, actividades que poseen un momento propio y tienen significado o intenciones para los que participan en ellas (Bronfenbrenner, 1987). Así, Bronfenbrenner concibe las prácticas como acciones intencionadas y reguladas que efectivamente asumen los adultos cuidadores para favorecer el aprendizaje, el crecimiento y el desarrollo de los niños.

Cada microsistema como contexto de desarrollo tiene un entorno físico y unas modalidades de relaciones proximales. No obstante, es importante destacar que son las relaciones las que dejan huella en el curso del desarrollo. Así, en la medida que la estabilidad de las relaciones se hace más fuertes y la variabilidad de los ambientes aumenta, entonces los procesos proximales tienen efectos significativos en las trayectorias de vida. Desde esta perspectiva, las actividades y las relaciones entre los microsistemas como contextos de interacción educativos enmarcan el mesosistema y los cambios significativos son analizados en función de transiciones ecológicas entre microsistemas, es decir, en la mutua acomodación entre el individuo y sus ambientes educativos primarios (Bronfenbrenner, 1993). Para este autor, las transiciones ecológicas tienen lugar "cuando la posición de una persona en el ambiente ecológico se modifica como consecuencia de un cambio de rol, de entorno, o de ambos a la vez" (Bronfenbrenner, 1987, p. 46). Este aspecto es relevante porque muestra la incidencia de las transiciones en el desarrollo del sujeto.

Los contextos culturales tienen un entorno físico, en el cual interactúan actores en el curso de la realización de actividades, movidos por propósitos y metas, y que tratan de comunicarse y negociar unos con otros. Las variaciones se presentan en los entornos físicos, los actores que intervienen, sus vínculos interpersonales, sus intenciones, sus metas, el tipo de actividades socioculturales que realizan y los procesos de negociación que llevan a cabo.

En los estudios acerca de la interacción de niños preescolares y sus cuidadores se proponen las siguientes dimensiones como fuentes de influencia de los logros del desarrollo de niños pertenecientes a minorías: creencias culturales, prácticas de crianza, condiciones de salud, prácticas de cuidado de la 
salud, factores biológicos, procedencia étnica y prácticas educativas. Entre tanto, otros indicadores de la incidencia del contexto en el desarrollo pueden ser las siguientes dimensiones: estructura familiar, características de los padres y/o del adulto que cuida del niño, estado de empleo de los padres, condiciones de la vivienda, condiciones sociales y económicas generales de la familia, acceso a servicios básicos y sanitarios, prácticas de crianza relativas a la salud, la alimentación y la socialización, las modalidades de regulación del comportamiento, y la prevención de situaciones de maltrato o de abuso en la familia. Sin embargo, se encontraron pocas evaluaciones acerca de las competencias de las familias y los programas educativos que trabajan con primera infancia (Orozco, Perinat \& Sánchez, 2009; Orozco, Sánchez \& Cerchiaro, 2012).

Estas aproximaciones teóricas y empíricas exponen la problemática de la dicotomía sujeto/contexto, pero no resuelve la compleja relación sujeto - contextos culturales específicos de interacción. La evidencia empírica muestra que los niños pequeños construyen conocimiento en la familia, en el hogar comunitario, en la escuela y en la comunidad, no obstante, el objetivo central de estos estudios se concentra en tipificar los contextos de interacción para describir el desarrollo de los niños. Al respecto de esta postura, asumimos un distanciamiento teórico y metodológico del modelo asociacionista que establece relaciones lineales entre contextos de interacción, actividades y desarrollo cognitivo.

Otra tendencia indaga específicamente acerca de las capacidades, las habilidades y las destrezas de los niños desde una perspectiva centrada en el desarrollo del individuo. En esta perspectiva, los contextos culturales específicos son considerados variables que tienen alguna incidencia en el desarrollo de los niños, pero no son determinantes en la equilibración progresiva de estructuras cognitivas (Piaget \& Inhelder, 1976) o en las cartografías mentales de dominio específico que permiten a los niños preescolares construir conocimiento (Karmiloff, 1994; Lee, 2000).

La conceptualización en este campo de conocimiento revela que el desarrollo cognitivo se ha movido entre la polaridad: capacidades de dominio general y capacidades de dominio específico. Para los piagetianos, el desarrollo implica la construcción de estructuras generales, comunes a todos los dominios, que operan sobre el sistema cognitivo de manera similar. Los cambios en las estructuras lógicas dan lugar a la sucesión de estadios. En términos metodológicos, la escuela piagetiana diseñó un conjunto de tareas "engañosas" de resolución de problemas que permiten acompañar los argumentos y las acciones de los niños que tienen como punto de partida el desarrollo de perspectivas perceptuales centradas en argumentos acerca de las relaciones directas de los objetos hasta múltiples perspectivas con argumentos que se descentran del dato perceptivo para reconstruir operacionalmente las relaciones de los objetos.

En las perspectivas de dominio específico, se observa el cambio en el desarrollo a partir de procesos graduales de transformación de procedimientos, que van desde la automatización del conocimiento con una menor accesibilidad hasta procesos de "explicitación" con accesibilidad cada vez mayor, lo que implica "representar explícitamente la información implícita en las representaciones procedimentales en que se apoya la estructura de la conducta" (Karmiloff, 1994, p. 36).
En esta perspectiva, los análisis de la psicología del desarrollo se centran en fenómenos y datos que permiten entender que el acceso al conocimiento supone conocimientos cualitativamente distintos a los que se desprenden de la visión lineal y aséptica de una concepción por etapas. Más que una secuencialidad y un orden, "el acceso y el paso de un conocimiento a otro parece marcado por fenómenos tales como el de la variabilidad" (Puche, 2003, p. 20), en tres modalidades diferenciadas: las estrategias intrasujeto, la capacidad de representar el mismo conocimiento en formatos implícitos y explícitos, y finalmente, la variabilidad intragrupo, o sea, la variabilidad de respuestas y estrategias en un mismo grupo de edad al resolver un problema (Puche, 2003).

Desde la década de los noventa, emergió un enfoque teórico y metodológico que busca explicar la actividad cognitiva cambiante y la variabilidad del desempeño de los sujetos como un sistema dinámico que puede definirse en distintos niveles de complejidad (van Geert, 1998). En esta perspectiva, la temporalidad juega un papel fundamental en la reconstrucción dinámica de los procesos psicológicos, los cuales son analizados no como estados estáticos sino como procesos en cambio permanente. Por lo tanto, esta perspectiva busca comprender cómo se dan los cambios, más que establecer qué cambia (Puche, 2009; Guevara, 2015). Así, se destaca el trabajo de Paul van Geert acerca del desarrollo del lenguaje y la conceptualización teórica de los sistemas dinámicos aplicados a la actividad psicológica, los estudios de razonamiento científico en niños pequeños (Guevara, van Dijk, \& van Geert, 2016) o las interacciones madre-niño y su incidencia en el desarrollo afectivo en el primer año de vida (Sánchez \& Cuadros, 2014).

Este enfoque, centrado en los sistemas dinámicos no lineales, ha contribuido enormemente a la comprensión del desarrollo desde la perspectiva de la complejidad (Valsiner, 2007). No obstante, surge un desafío por resolver: entregar nuevas opciones de modelamiento matemático-formal a partir de modalidades de análisis centradas en el estudio de caso, para superar las relaciones lineales de las inferencias inductivas en las estadísticas convencionales y conseguir la comprensión del desarrollo desde la complejidad, a partir de la generalización de los procesos psicológicos.

El enfoque sistémico es el eje central de la tercera tendencia, en el cual, la persona es un sistema abierto. Por lo tanto, lo importante no son las unidades que describen la persona, sino las relaciones que definen el sistema. Así un aspecto fundamental es traer el concepto de organización para caracterizar estas relaciones mutuas:

El niño es un sistema abierto. Un sistema es una unidad compleja en el tiempo y en el espacio. El término unidad remite a que posee unos límites o fronteras que lo separan/diferencian de todo lo que no es él. La noción de complejidad que se predica de un sistema se ha de entender como que es posible distinguir en él, "partes" que están en mutua relación y que esta última puede ser difícil de explicar específicamente. La manera como las partes de un sistema y sus relaciones contribuyen a su existencia es su organización. Mantener esta última comporta una dinámica interna. Un sistema es abierto, además, cuando realiza intercambios con lo que existe afuera de él; tales intercambios también contribuyen a que perdure su organización (Perinat, 2003, p. 48-49). 
Este enfoque permite establecer relaciones entre los procesos psicológicos que los niños utilizan al participar en prácticas de su comunidad y sus contextos educativos de interacción. Desde esta perspectiva teórica para abordar el desarrollo de los niños como un sistema abierto y complejo, es necesario tipificar el desarrollo cognitivo y caracterizar los contextos de interacción para establecer relaciones posibles entre ambas dimensiones de problema:

Para esta tendencia, el desarrollo está vinculado a los cambios que operan en el sujeto, y le permiten construir progresivamente diferentes tipos de capacidades, es decir, estructuras con modalidades de funcionamiento afectivo, cognitivo y social para responder a las demandas de su contexto. Los mecanismos de auto-regulación emergen de la experiencia del sujeto a partir de su participación en prácticas concretas de su comunidad (Orozco, Perinat \& Sánchez, 2009, p. 289).

En términos metodológicos, estos estudios utilizan prácticas culturales de comunidades específicas para observar y comprender el desarrollo de los niños. Simultáneamente, utilizan estrategias de análisis microgenético para describir los desempeños de los niños en función de las estrategias que utilizan para equivocarse o para acertar en la resolución de las tareas: se analizan las habilidades y las destrezas con el propósito de hacer visible lo que los niños saben hacer y pueden decir (Orozco, et al, 2009; Orozco, et al 2012).

En esta tendencia, la inferencia, como proceso psicológico superior, emerge como un modo de articulación simbólica que permite nuevas formas de autoorganización personal a partir de experiencias concretas en contextos culturales específicos. Por lo tanto, se asume que la inferencia implica la adopción consciente y controlada de una creencia como consecuencia de otros conocimientos (Sánchez, 2017). Así, el razonamiento inferencial se apoya en la representación mental de relaciones entre entidades para crear evidencias que configuran otras entidades. La inferencia como proceso cognitivo, permite ir más allá de la información dada para generar una nueva conclusión, formar una generalización o encontrar una solución para un problema que tiene como resultado, generalmente, nuevo conocimiento (DeLoache, Miller \& Pierroutsakos, 1998).

El racionamiento inferencial permite reflexionar, no solo por lo que es, sino también por lo que puede ser, por lo que debe ser o por lo que tiene que ser. Es posible argumentar que si un hecho es cierto, entonces, otra cosa sobre la cual no tenemos evidencia inmediata puede -tiene o debe- ser cierta también. De esta manera, la inferencia es inmediata, en la medida en que identificamos el cambio y sencillamente formulamos el nuevo enunciado con fluidez y probablemente con eficacia. Aunque, la inferencia puede utilizar racionamientos probabilísticos simples porque están fundamentados en las posibilidades de ocurrencia, también se tornan complejos porque están al servicio de la racionalidad científica o de sofisticados racionamientos sociales.

En este enfoque sistémico, la inferencia o acción de inferir, emerge como un proceso afectivo-cognitivo que surge en la relación con el otro y con el mundo, para establecer relaciones entre posibilidades de un evento futuro a partir de aspectos específicos que acontecen en el presente. Así, el carácter proyectivo de este proceso admite la objetivación del mundo a partir de la subjetivación del otro. En este sentido, los procesos inferenciales actúan como puerta de entrada al crear un sistema anticipatorio que permite formular hipótesis que pueden (o no) ser correctas sobre las potencialidades y límites de la relación yo-otro-mundo (Valsiner, 2007). Como proceso, la inferencia permite observar el cambio en el desarrollo ontogenético, y simultáneamente permite reflexionar acerca de los procesos semióticos que emergen en la relación dialógica yo-otro-mundo. Esta última característica es el foco de interés del enfoque sistémico.

\section{Método}

Este es un estudio exploratorio descriptivo acerca de las relaciones posibles entre el desarrollo cognitivo de niños y sus contextos educativos de interacción en la modalidad Hogares Comunitarios del ICBF (HC-ICBF) ${ }^{3}$.

\section{Participantes}

En este estudio participaran 405 niños: 134 niños de 3 años, 136 niños de 4 años y 135 niños de 5 años que viven en sectores urbanos pobres. De igual manera, participaron 96 madres comunitarias que atienden los HC-ICBF a los cuales asisten los niños. La selección de las madres atendió a procesos previos de formación a agentes educativos. La selección de los niños atendió a los dos criterios: asistencia al programa HCICBF y edad.

\section{Instrumentos y modalidad de análisis}

Nuestro desafío fue utilizar métodos mixtos para comprender la complejidad de la relación entre el desarrollo cognitivo de los niños y sus contextos educativos de interacción en la modalidad Hogares Comunitarios del ICBF (HC-ICBF).

Para indagar acerca del desarrollo cognitivo utilizamos una Situación de Resolución de Problemas (SRP) que tuvo por objetivo buscar tres personajes dibujados sobre un tablero, completamente ocultos por tarjetas removibles. En la presentación del problema se siguió este procedimiento: inicialmente, se cualificó a los agentes educativos para presentar la SRP, así el entrevistador presentó las siluetas de personajes que vestían atuendos relativos a la siguiente actividad: personaje nadando $(A)$, personaje volando $(B)$ y personaje corriendo $(C)$. El tablero con tarjetas removibles tenía imágenes de animales que realizaban una actividad semejante a la actividad del personaje y entornos relativos a la actividad de cada personaje. Entonces en la tarea, dos tipos de indicios de actividad se presentaban: dibujos de animales con actividades que corresponden a la actividad de cada personaje: peces (A1), pájaros (B1) y caballos (C1). Dibujos de los entornos que posibilitan cada tipo de actividad: olas (A2), nubes (B2) y prado (C3). La ubicación de ambos indicios (1 y 2) corresponde con la ubicación de cada personaje oculto: personaje nadando $(A, 1,2)$; personaje volando $(B, 1,2)$ y personaje corriendo $(C, 1,2)$.

El propósito de la SRP es encontrar a cada personaje oculto en uno de los cuatro cuadrantes del tablero. Para resolver el problema, el niño debe descubrir al personaje ( $A, B$ y $C$ ) a partir de los indicios (1 y 2) en cada cuadrante del tablero. Entonces, el niño tiene que inferir la relación de semejanza entre la actividad del personaje en la silueta con la actividad de los animales en cada cuadrante (A1, B1 y C1), inferir la relación de 
semejanza entre la actividad del personaje en la silueta con los entornos (A2, B2 y C2) o inferir la relación de semejanza entre la actividad del personaje en la silueta con ambos indicios ( $A$ 1, 2; B, 1, 2 y C 1, 2). Esta modalidad de tareas permite explorar los procesos psicológicos superiores que los niños despliegan en la resolución y seguir la trayectoria de su pensamiento a partir de las estrategias que subyacen a su desempeño. Se utilizaron dos niveles de análisis para obtener un indicador del desarrollo cognitivo de cada niño, a saber: el primer nivel identifica su desempeño: no identifica personajes, identifica un personaje, dos o tres personajes ocultos detrás del tablero. El segundo nivel especifica la estrategia de búsqueda que involucra el uso de la inferencia en la resolución del problema. La Tabla 1 presenta el conjunto de estrategias identificadas para la búsqueda del personaje.

Tabla 1. Descripción de estrategias de búsqueda

\begin{tabular}{cl}
\hline Nivel & \multicolumn{1}{c}{ Estrategia de búsqueda } \\
\hline 1 & Búsqueda al azar \\
2 & Búsqueda al azar limitada a un cuadrante \\
3 & Búsqueda al azar y reconocimiento de indicios 1 \\
4 & Búsqueda al azar y reconocimiento de indicios 2 \\
5 & $\begin{array}{l}\text { Búsqueda por indicios } 1 \text { y } 2 \text { para el reconocimiento } \\
\text { del personaje }\end{array}$ \\
\hline
\end{tabular}

En la indagación acerca de los contextos educativos se utilizó una entrevista semiestructurada que permite la caracterización de los agentes educativos, las expectativas de su trabajo y las redes de apoyo. La Tabla 2 presenta una relación entre dimensiones e indicadores del HC-ICBF que se indagaron en la entrevista.

Tabla 2. Dimensiones e indicadores que describen el HC-ICBF con la entrevista

\begin{tabular}{cl}
\hline \multicolumn{1}{c}{ Dimensiones } & \multicolumn{1}{c}{ Indicadores } \\
\hline $\begin{array}{c}\text { Caracterización } \\
\text { del agente } \\
\text { educativo }\end{array}$ & Edad, origen, nivel educativo, formación \\
\hline Expectativas & $\begin{array}{l}\text { Cualidades y valores que pretenden } \\
\text { formar en los niños para el futuro }\end{array}$ \\
& $\begin{array}{l}\text { Nivel de formación educativo y ejercicio } \\
\text { laboral de los niños en el futuro }\end{array}$ \\
& $\begin{array}{l}\text { Acciones que implementa día a día } \\
\text { para conseguir las cualidades, valores, } \\
\text { nivel de formación educativa y ejercicio } \\
\text { laboral de los niños después de egresar } \\
\text { del HC-ICBF }\end{array}$ \\
\hline Redes de apoyo & $\begin{array}{l}\text { Redes de apoyo familiar en el HC-ICBF } \\
\text { Redes de apoyo de padres usuarios en } \\
\text { el HC-ICBF } \\
\text { Redes de apoyo comunitario en el HC- } \\
\text { ICBF }\end{array}$ \\
\hline
\end{tabular}

La observación de trabajo de campo permitió identificar las siguientes dimensiones que describen los HC-ICBF: prácticas de protección y cuidado, prácticas de formación, prácticas de entretenimiento, prácticas de regulación del comportamiento. La Tabla 3 presenta la relación entre dimensiones e indicadores que permitieron caracterizar el HC-ICBF a partir del uso de la observación.

Tabla 3. Dimensiones e indicadores que describen el HC-ICBF a partir de la observación

\begin{tabular}{|c|c|}
\hline Dimensiones & Indicadores \\
\hline $\begin{array}{l}\text { Prácticas de } \\
\text { protección y } \\
\text { cuidado }\end{array}$ & $\begin{array}{l}\text { Esquema de vacunación completo, } \\
\text { promoción de la salud, protección } \\
\text { frente al maltrato físico y el abuso, } \\
\text { prácticas alimentación cotidianas }\end{array}$ \\
\hline \multirow{3}{*}{$\begin{array}{l}\text { Prácticas de } \\
\text { formación }\end{array}$} & $\begin{array}{l}\text { Actividades de formación para la vida } \\
\text { cotidiana }\end{array}$ \\
\hline & $\begin{array}{l}\text { Actividades de formación para la es- } \\
\text { cuela }\end{array}$ \\
\hline & $\begin{array}{l}\text { Actividades de formación para la vida } \\
\text { en comunidad }\end{array}$ \\
\hline $\begin{array}{l}\text { Prácticas de } \\
\text { Entretenimiento }\end{array}$ & $\begin{array}{l}\text { Prácticas lúdicas relacionadas con el } \\
\text { juego, la música, la danza, el teatro, el } \\
\text { dibujo, la pintura, la literatura }\end{array}$ \\
\hline \multirow{3}{*}{$\begin{array}{l}\text { Prácticas de } \\
\text { Regulación del } \\
\text { comportamiento }\end{array}$} & $\begin{array}{l}\text { Límites y prohibiciones para el niño } \\
\text { en el HC-ICBF }\end{array}$ \\
\hline & Cumplimiento de las prohibiciones \\
\hline & $\begin{array}{l}\text { Actividades correctivas frente al in- } \\
\text { cumplimiento de los límites y las pro- } \\
\text { hibiciones }\end{array}$ \\
\hline
\end{tabular}

Las dimensiones permiten caracterizar los HC-ICBF. Cada dimensión está estructurada por indicadores, los cuales se indagaron a partir de preguntas o descriptores en la observación de campo. De esta manera, los indicadores se operacionalizaron en variables. Para la transformación de los indicadores en variables se utilizó el siguiente procedimiento: 1) valoración y categorización de las respuestas y los descriptores observacionales en función de criterios de favorabilidad o desfavorabilidad para el desarrollo del niño convenidos entre los agentes educativos y el investigador; 2) sumatoria de los valores acordados para cada variable y 3) categorización de la variable en niveles de acuerdo con los percentiles obtenidos a partir de la distribución de la población. Generalmente se identificaron entre 3 y 4 niveles. Así, cada dimensión está compuesta por diferentes variables. El puntaje final para cada dimensión se obtuvo a partir del nivel alcanzado en la transformación de cada variable: sumatoria de los niveles obtenidos en las variables componentes de la dimensión, posteriormente, obtención del porcentaje correspondiente al nivel alcanzado por cada HC-ICBF en función del puntaje máximo posible para cada dimensión, y por último, la asignación del puntaje para cada dimensión en función de los rangos encontrados, definidos por los percentiles resultante de la distribución de la muestra. Los puntajes definen los niveles que cada HC-ICBF alcanza en cada dimensión.

Este estudio utilizó métodos mixtos de carácter secuencial ${ }^{4}$ con predominancia de la parte cualitativa para el diseño de instrumentos y la elaboración de categorías analíticas que,

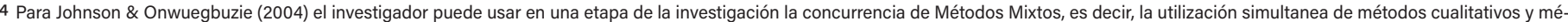

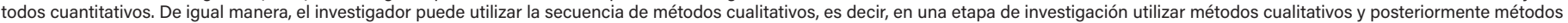
cuantitativos (o viceversa). Para el caso de esta investigación se utilizó Métodos Mixtos de Carácter Secuencial con Igualdad en el estatus: Cual $\rightarrow$ Cuan. 
posteriormente, se transformaron en datos cuantitativos. El uso de estadísticas no paramétricas, análisis de correspondencias múltiples (ACM), permitió adoptar los datos cuantitativos para establecer relaciones no lineales entre el desarrollo cognitivo de los niños y sus contextos educativos para abordar la complejidad del desarrollo psicológico en contextos. Así, el ACM permitió analizar patrones de relaciones de diversas variables, de tipo nominal, para crear niveles diferenciados que dan cuenta del desarrollo cognitivo de los niños a partir del tipo de proceso inferencial que utilizan para resolver el problema y niveles para cada una de las dimensiones que describen los HC-ICBF. El objetivo del ACM es lograr la mayor información de un número grande de variables que se organizan en dimensiones (en nuestro caso, niveles de resolución de un problema y caracterización de los HC-ICBF). Esta técnica estadística se utiliza para analizar las relaciones de dependencia e independencia de un conjunto de variables categóricas a partir de los datos de una tabla de contingencia. Para ello se asocia a cada una de las modalidades de la tabla, un punto en el espacio $R n$ (generalmente $n=2$ ) de manera que las relacio- nes de cercanía/lejanía entre los puntos calculados reflejen las relaciones de dependencia y semejanza existentes entre ellas. Sea $X$ / Y dos variables categóricas, respectivamente, con valores $\{x 1, \ldots, x 8\}$ e $\{y 1, \ldots, y 8\}$. Se observan dichas variables en $\mathrm{N}$ elementos de una población. La intersección entre una fila y una columna da lugar a una celda o casilla, cuya frecuencia observada es Nij. En el ACM la información se organiza desde un punto de vista gráfico (plano cartesiano). De esta manera, entre más próxima estén las variables categoriales al eje, es decir, más cercanas a cero, su aporte a la explicación de la relación es menor.

\section{Resultados}

La presentación de los resultados del desarrollo cognitivo de los niños incluye el tipo de estrategias que utilizan en función de su edad al resolver la tarea. La Tabla 4 muestra la distribución del porcentaje de niños según la estrategia que utilizan en cada búsqueda, en función de la edad.

Tabla 4. Distribución del porcentaje de niños según el tipo de estrategia en función de la edad

\begin{tabular}{lccccccccc}
\hline Nivel & \multicolumn{3}{c}{ 3 años } & \multicolumn{3}{c}{4 años } & \multicolumn{3}{c}{5 años } \\
\hline 1 & B1 & B2 & B3 & B1 & B2 & B3 & B1 & B2 & B3 \\
2 & 19,4 & 20,9 & 15,7 & 21,3 & $18,4 /$ & 13,2 & 20,7 & 20,7 & 11,1 \\
3 & 21,6 & 14,2 & 4,5 & 19,9 & 15,4 & 2,9 & 16,3 & 9,6 & 2,2 \\
4 & 16,4 & 9,7 & 0,7 & 8,8 & 9,6 & 0,7 & 5,9 & 5,9 & 0,7 \\
5 & 17,2 & 38,1 & 50,0 & 24,3 & 30,1 & 50,7 & 23,0 & 25,9 & 31,1 \\
\hline
\end{tabular}

El análisis del porcentaje de los niveles de estrategias de búsqueda muestra que en todas las edades hay un incremento del uso de estrategias de nivel 4 y $5^{5}$ : a los 3 años, $42.6 \%$ utiliza ambos niveles de estrategias en la búsqueda del primer personaje; el $55.3 \%$, en la búsqueda del segundo personaje y el $79.1 \%$, en el tercer personaje. A los 4 años, el $50.0 \%$ utiliza ambos niveles en la búsqueda del primer personaje; $56.6 \%$, en la segunda búsqueda y el $83.1 \%$, en la tercera. A los 5 años, $57.1 \%$ las utilizan en la primera búsqueda, $63.7 \%$, en la segunda y $85.9 \%$, en la tercera. El mismo análisis aplicado a cada búsqueda muestra que los porcentajes de éxito igualmente difieren en función de la edad.

Un ANOVA de dos vías de clasificación permitió encontrar diferencias significativas en los porcentajes de niños que usan la estrategia de nivel 4 y la estrategia de nivel 5 , y aquellos que utilizan las estrategias regidas por el azar (N1, N2 y N3), en todas las edades, a través de las búsquedas. Para los 3 años $F(2,401)=23,78, p<.001$; para 4 años $F(2,407)=18,94, p<.001$; para 5 años $F(2,404)=14,17 p<.001$. La utilización de un ANOVA de dos vías de clasificación permitió igualmente encontrar diferencias significativas en los porcentajes de utilización de un tipo de estrategias, en función de la edad, a través de las tres búsquedas. Los niños de 4 años presentan variaciones significativas entre la estrategia de nivel 4 con respecto a las demás estrategias $F(4,14)=5.89, p<.05$; y los de 5 años, entre la estrategia de Nivel 5 y las demás estrategias $F(4,14)=14.92$, $p<.001$. No se encontró diferencia significativa entre las estrategias que utilizan los niños de 3 años.

En los tres grupos de edad encontramos que los niños mejoran significativamente su ejecución durante la resolución del problema: los porcentajes de éxito aumentan proporcionalmente de la primera a la tercera búsqueda, al igual que incrementa el uso de estrategias del más alto nivel relacional. Esto nos permite proponer que a medida que resuelven la SRP los niños logran centrarse más en la misma, lo que les permite reorganizar sus esquemas de acción y mejorar sus estrategias y, por ende, su desempeño. La distribución de los porcentajes de niños de 3 y 4 años, en función del número de personajes que encuentran es cercana a la curva normal, este dato permite señalar que la población de este estudio presenta un desempeño normal, probablemente similar al de cualquier otro tipo de población con la que se pueda trabajar este tipo de SRP. El desempeño de los niños de 5 años muestra una tendencia al éxito en las tres búsquedas a partir del uso de estrategias de los niveles 4 y 5 . Así, los niños de 5 años presentan una ejecución más exitosa que los de 3 y 4 años. Entonces, en la medida que la edad aumenta, los niños tienden a utilizar estrategias más elaboradas y a alcanzar un mejor desempeño. 
Sin embargo, en cada grupo de edad se presenta una gran variabilidad en las estrategias que utilizan en las tres búsquedas, o sea en la ruta que cada niño sigue.

Estos resultados permiten ver las dos caras del desarrollo: el desarrollo escalonado en función de la edad y la variabilidad en las ejecuciones en cada grupo de edad. A medida que la edad aumenta, la ejecución de los niños al resolver la tarea mejora. Este hecho nos indica que la tarea utilizada permite describir el desarrollo cognitivo de los niños y que su desarrollo presenta una tendencia normal y en algunos casos alta. Pero en cada nivel de edad se presenta una gran variabilidad en las estrategias que utilizan los niños, variabilidad que se evidencia cuando analizamos las estrategias y no el desempeño y que es generada por la variación intraindividual. Esta variabilidad se puede entender en dos sentidos: resultados diferenciados del mismo sujeto a través de las tres búsquedas, que se expresa en la variabilidad de las estrategias y resultados diferenciados dentro del mismo grupo de edad. Actualmente, la psicología del desarrollo cognitivo describiría este fenómeno como un caso típico de variabilidad en la ejecución, que da cuenta del cambio cognitivo.

Un alto porcentaje de agentes educativos que trabajan en los $\mathrm{HC}$-ICBF se ubican en el rango de edad entre 31 y 50 años. $28 \%$ de madres comunitarias no han cursado el grado noveno, nivel educativo mínimo que exige el programa HC-ICBF para ejercer el trabajo. Un grupo significativo de madres son mayores de 60 años y no han cursado la secundaria completa, dos aspectos que es necesario revisar en el proceso de selección y cualificación de los agentes educativos que trabajan con la primera infancia. Aunque resaltamos que $97 \%$ asiste a las actividades de capacitación programadas por el ICBF.

En la caracterización de los HC-ICBF es posible identificar tres modalidades de prácticas cotidianas que organizan la propuesta educativa en el HC-ICBF:

Prácticas de cuidado y protección. Respecto de estas prácticas destacamos que $100 \%$ de los HC-ICBF presentan el registro civil de los niños; $94 \%$ de las madres comunitarias realizan el control de talla y peso; $86 \%$ de los HC-ICBF visitados cumplen con las condiciones de higiene establecidas para prestar el servicio de cuidado; $72 \%$ cumplen con la minuta alimenticia establecidas por el programa; $60 \%$ presentan el registro del esquema de vacunación completo; $31 \%$ de las madres comunitarias admiten que pueden reconocer situaciones de maltrato infantil. Este grupo de madres utiliza dos estrategias de intervención: establecen contacto con los padres (87\%) y reportan el caso a profesionales del programa HC-ICBF (16, $9 \%)$.

Los resultados muestran el buen desempeño de las madres en el control de talla y peso, aspecto central en la promoción de un adecuado crecimiento de los niños. En las prácticas relacionadas con la alimentación, las madres reportan que utilizan la minuta que establece el programa HC-ICBF como referente en la preparación de los alimentos, solo un grupo reducido de madres reciben colaboración en esta actividad. Por lo tanto, las madres comunitarias en una jornada diaria atienden la cobertura de alimentación en desayunos, almuerzos y refrigerios que sustituye las comidas que los niños reciben en sus casas. Las prácticas relacionadas con higiene y aseo tienen una presencia significativa en la vida cotidiana de los niños y son parte de un plan preventivo de la salud: bañarse, limpiarse los dientes y lavarse las manos. Aunque existe un esfuerzo por promover el seguimiento al esquema de vacunación, el control visual y auditivo, su monitoreo no es una constante en todos los HC- ICBF que participaron en el estudio. Sin embargo, podemos afirmar que las prácticas de cuidado y protección de las madres comunitarias están garantizando la supervivencia de los niños que viven en sectores urbanos pobres de Colombia. No obstante, es pertinente formular las siguientes preguntas: ¿Cuál es el tiempo real que emplea la madre comunitaria para el cuidado y protección de los niños? ¿Estas actividades concentran el trabajo de atención del agente educativo durante una jornada de trabajo? ¿Qué otro tipo de estrategias se pueden establecer para ampliar el espectro de actividades?

Prácticas de formación. 93\% de los agentes educativos enseñan prácticas de la vida cotidiana relacionadas con la higiene y el aseo, también actividades para los asuntos de la vida cotidiana: aprender a amarrarse los zapatos, a decir el nombre de los papás, a recordar el teléfono de la casa, a decir la dirección del HC-ICBF. 70\% realizan actividades de formación orientadas a escolarización: aprender las letras, los números, los colores, las figuras geométricas.

Prácticas de entretenimiento. En el 96\% de los HC-ICBF se realizan actividades lúdicas como salir al parque, escuchar música, ver películas, cantar y bailar. En esta modalidad de prácticas se destaca un alto porcentaje de madres comunitarias que realizan actividades lúdicas en el HC-ICBF. Los juegos de reglas tradicionales, los relatos, las danzas, la literatura infantil y la visita a centros deportivos, son algunos de los escenarios que privilegian las madres comunitarias para promover la educación de los niños.

Prácticas de regulación del comportamiento. 98\% de las madres tienen un sistema de regulación del comportamiento de los niños para evitar la violencia entre pares. Sin embargo, solo $68 \%$ de las madres manifiesta que los niños cumplen estas reglas. En esta modalidad de prácticas de formación son frecuentes formas de regulación del comportamiento como sistemas de reglas, prohibiciones y correcciones $y$, usualmente, los niños cumplen las reglas. Si no acontece el cumplimiento, el agente educativo corrige al niño para que cumpla las reglas del HC-ICBF. Sin embargo, el sistema regulativo no emerge de acuerdos entre el agente educativo, los padres de familia y los niños. Por lo tanto, es necesario verificar si las estrategias de corrección resultan ser las más adecuadas y oportunas para generar respeto y responsabilidad. Las madres reportan que detectan el maltrato de los niños en su casa. En las entrevistas, comentan que la estrategia más utilizada para identificar cualquier tipo de maltrato son las conversas con el niño acerca de asuntos familiares y también, huellas físicas del maltrato. En cuanto a las acciones que emprende la madre comunitaria frente a situaciones de maltrato, resulta de especial interés para este estudio, encontrar un bajo porcentaje de reportes de casos ante las autoridades competentes.

Destacamos las expectativas de los agentes educativos. Más del $60 \%$ de las madres comunitarias esperan que los niños al salir del HC-ICBF posean cualidades que les permitan ser miembros respetados por su comunidad. Sin embargo, un grupo significativo de madres no encuentra relevante "especular sobre el futuro de los niños". 
Utilizamos el modelo de ACM para relacionar los indicadores de desarrollo cognitivo y los indicadores que caracterizan el HC-ICBF. El modelo revela que diferentes niveles de desarrollo de la inferencia presentan relación con algunas variables que describen el contexto educativo. De esta manera, niños de los diferentes grupos de edad que usan estrategias de inferencia de nivel 4 y 5 pertenecen a HC-ICBF, en los que predominan porcentajes altas de prácticas de regulación del comportamiento, formación, entretenimiento, protección y cuidado. En contraste, niños de los diferentes grupos de edad que utilizan estrategias de inferencia 1 y 2 , pertenecen a $\mathrm{HC}$ ICBF con porcentajes bajos de prácticas de formación y expectativas bajas. La Figura 1 presenta las relaciones posibles.

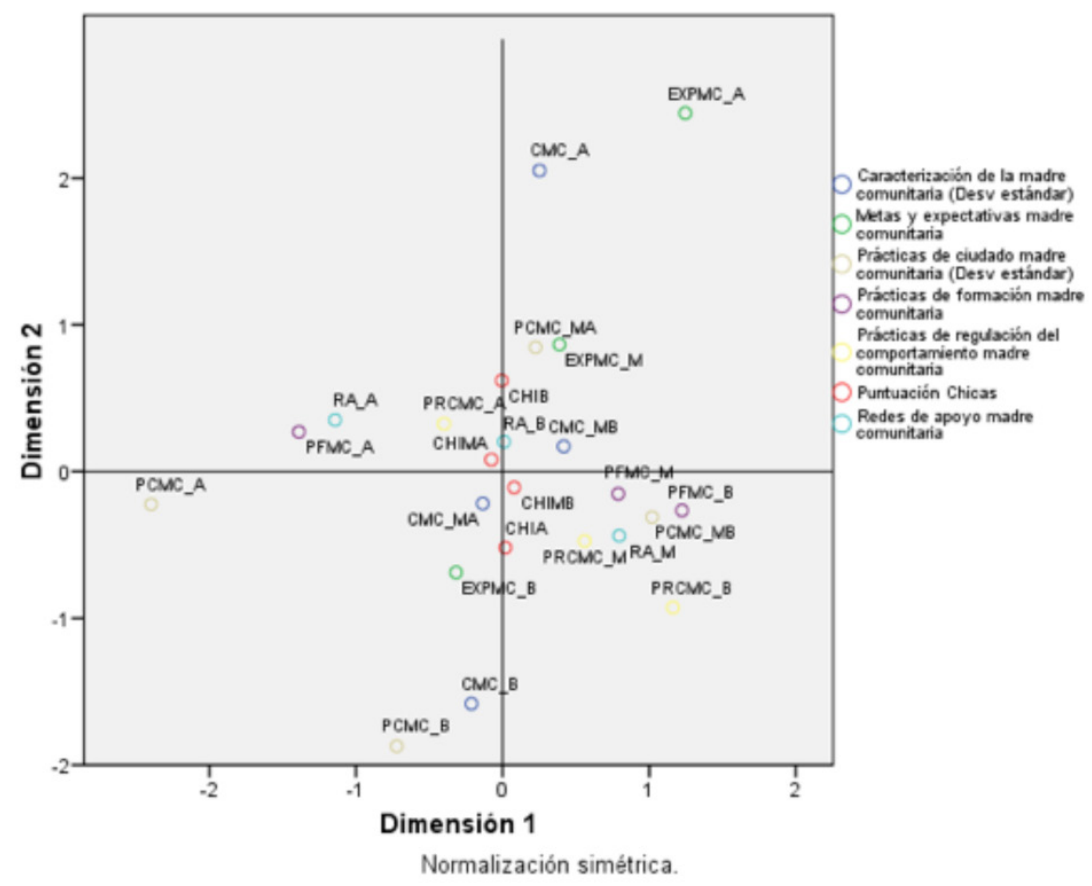

Figura 1. ACM relación entre el uso de la inferencia y HC-ICBF

De esta manera, el ACM destaca una fuerte relación entre las metas y expectativas altas de los agentes educativos con las estrategias complejas de resolución de niños de diferentes grupos de edad. Las variables edad y nivel de escolarización del agente educativo se relacionan con las prácticas de formación para la escuela. Las prácticas de formación para los asuntos de la vida cotidiana tienen una significativa relación con altas redes de apoyo. De esta manera, se destaca la relación significativa entre los niveles de desarrollo de la inferencia con las expectativas que tienen los agentes educativos de los HC-ICBF.

El propósito central es presentar relaciones entre indicadores del desarrollo cognitivo e indicadores que describen los contextos educativos. El ACM establece que los cinco niveles que describen del uso de la inferencia en los niños al resolver una tarea tienen una relación significativa con las expectativas de los agentes educativos respecto al futuro de los niños que asisten al HC - ICBF. Destacamos que el ACM no establece relaciones lineales y deterministas entre las estrategias de inferencia de los niños y las variables que caracterizan los HC-ICBF. Aunque el modelo permite diferenciar los niveles de estrategias en la resolución del problema, en el momento de incorporar el conjunto de variables del contexto educativo, el modelo pierde en varianza explicativa. De esta manera, ACM muestra que las dimensiones del HC-ICBF no revelan relaciones tan fuertes con las variables que describen el desarrollo de los niños, tal como ocurrió en el análisis realizado con la interacción familiar (Orozco, et al, 2009; Orozco, et al, 2012) donde los resultados permiten establecer otras relaciones: altas expectativas del agente educativo se relacionan significativamente con las características que tienen las madres comunitarias al respecto de su edad y su nivel de escolarización. Además, prácticas de formación altas en el HC-ICBF se relacionan con altas redes de apoyo.

\section{Discusión}

Este artículo se inscribe en un diálogo de frontera entre el enfoque sistémico de la psicología del desarrollo y la educación para la primera infancia. El propósito central son las relaciones entre la emergencia de procesos psicológicos superiores, como la inferencia, que utilizan los niños al participar en SRP anclados, y sus contextos educativos de interacción en la modalidad de HC-ICBF.

La presencia simultánea en el HC-ICBF de un conjunto de prácticas de cuidado, protección y formación en la jornada de trabajo de la madre comunitaria permite fortalecer el sentimiento y las expectativas que en los sectores urbanos pobres es posible ofrecer mejores opciones para la educación de los niños pequeños. Para el enfoque sistémico, los procesos intrasubjetivos son individuales, pero a la vez se construyen en contextos específicos como procesos interpsicológicos (Valsiner, 2007). Para esta perspectiva la internalización y la externalización de la acción simbólica se construye en un sistema complejo de la relación yo otro mundo (Sánchez, 2017). Así, la acción simbólica que estructura el yo se construye en 
diferentes contextos: en la casa con la familia, en el HC-ICBF con la madre comunitaria y los compañeros, en el parque con los amigos de la comunidad. Por lo tanto, la acción del sujeto y sus contextos de interacción son indisociables en la construcción de la subjetividad (Perinat, 2003). Así, el individuo transforma con la acción subjetiva el ambiente físico y objetivo en ambiente percibido, pensado y sentido, esto es, en una construcción personal a ser compartida y negociada con otros individuos, igualmente constructores. Ese proceso de construcción del conocimiento de la realidad en el HC-ICBF ocurre no solo para el mundo material, más también para el mundo de las ideas, de las personas y del sí mismo (Simão \& Sanchez, 2017).

Las prácticas cotidianas de cuidado son acciones comunitarias para garantizar la supervivencia de las nuevas generaciones en los sectores urbanos pobres. Las prácticas de protección del programa HC-ICBF en Colombia buscan albergar a los niños mientras sus padres trabajan por fuera de sus casas. Estos hogares comunitarios son fronteras que se abren a las comunidades para acompañar a los niños en condiciones de vulnerabilidad. No obstante, los niños tienen barreras simbólicas que los exponen a los peligros del entorno y necesitan de la protección de los adultos. En actualidad, los padres de familia están tranquilos mientras salen a trabajar porque los niños están bajo la tutela de las madres comunitarias. Aunque, la impotencia emerge en las comunidades cuando tienen que enfrentar la presencia de agentes agresores que intimidan con la delincuencia y la violencia urbana. Bajo esas circunstancias las medidas de protección del HC-ICBF se reduce a impedir que los niños circulen en las calles expuestos a la violencia.

Las prácticas educativas son el espacio para los juegos, las danzas y las leyendas propias de la comunidad. En el espacio del HC-ICBF los niños participan en juegos de la tradición oral, bailan danzas tradicionales y bailes de temporadas, escuchan y cuentan historias de la tradición oral o relatos cotidianos. La presencia simultánea de este conjunto de prácticas educativas busca fortalecer el sentimiento de pertenecer a una comunidad.

Para esta perspectiva, la inferencia, como la acción simbólica que estructura el yo, emerge de la relación del sujeto con objetos y situaciones en sus diferentes contextos de interacción: la familia; el HC-ICBF, la escuela, el barrio y la comunidad durante las SRP, los relatos, los juegos y prácticas comunitarias. De esta manera, inferencia y contextos de interacción son indisociables en la construcción de la subjetividad de la acción.

Para la perspectiva sistémica, la persona en desarrollo transforma el ambiente físico en ambiente subjetivo, esto es, como una construcción personal a ser compartida y negociada con otros individuos, igualmente constructores (Perinat, 2003). Ese proceso de construcción del conocimiento de la realidad ocurre no solo para el mundo material, sino también para el mundo de las ideas, de las personas y del sí mismo.

De igual manera, es posible considerar que la inferencia en la relación yo-otro-mundo da la oportunidad a un conjunto de significaciones en el que ocurre una multiplicidad de reconstrucciones de mensajes compartidos. En ese proceso dialógico, cada persona transforma activamente las expresiones del otro, integrándolas en su base afectivo-cognitiva personal, proceso que transcurre en el ámbito de cultura personal. A la vez, este proceso hace referencia a la dinámica que ocurre en la preservación y transformación cultural, en que el otro se constituye en oportunidad para el yo de indagar y ser indagado por la tradición cultural, proceso de negociación en el ámbito de la cultura colectiva (Simão, 2010).

En esta relación emerge una concepción relacional desarrollista, en la cual el individuo está por descubrir las oportunidades y límites de la acción simbólica en los contextos educativos en los que participa (Ilámese familia, HC-ICBF o escuela) para utilizar posteriormente esas oportunidades y actuar sobre estos contextos de interacción a partir de la creación de nuevas posibilidades de acción para sí y para el otro. Por tanto, "al asir cognitiva y emocionalmente, el individuo construye significados que, en la dinámica de los significados construidos por otros actores, constituirán la cultura. Esta, a su vez, actuará como campo de acción" (Simão, 2010).

El estudio en la versión empírica inicial presenta una problemática vigente y relevante para la investigación en psicología del desarrollo. Esta perspectiva sistémica devela que los niños "piensan y piensan bien" y son capaces de utilizar complejas estrategias de inferencia, planificación, clasificación, anticipación y categorización al participar en prácticas culturales propias de su comunidad (Orozco, et al, 2009; Orozco, et al, 2012). No obstante, los elementos conceptuales que articula la perspectiva sistémica presentaron un paralelismo que nunca permitió encontrar un punto de intersección entre los aspectos centrales del estudio: procesos psicológicos superiores, contextos educativos y desarrollo psicológico de los niños. En el futuro se puede abordar este problema de investigación con métodos que aborden la complejidad, el dinamismo y la bidireccionalidad del desarrollo psicológico en el ciclo cultura - individuo - cultura a partir de nuevas reflexiones teóricas y metodológicas de la psicología cultural del desarrollo.

\section{Referencia}

Bronfenbrenner, U. (1987). La Ecología del Desarrollo Humano. Barcelona: Paidós

Bronfenbrenner, U. (1993). La educación de los niños en dos culturas. Madrid: Visor Editores.

Burchinal, M., Clifford, R., Culkin, M., Howes, C., Kagan, S., Peisner, E. \& Yazejian, N. (2001). The Relation of Preschool Child-Care Quality to Children's Cognitive and Social Developmental Trajectories through Second Grade. Society for Research in Child Development, 72 (5) 1534-1553

Calero, M., Mata, S., Carles, R., Vives, C., López S., Fernández A., \& Navarro, E. (2013). Learning potential assessment and adaptation to the educational context: the usefulness of the acfs for assessing immigrant preschool children. Psychology in the Schools, 50 (7), 705 - 721.

Carrol, B., Fuller, B., Loeb, S. \& Kagan, S. (2003). How Welfare Reform Affects Young Children: Experimental Findings from Connecticut: A Research Note. Journal of Policy Analysis and Management., 22 (4) 537 550.

Carrol, B., Fuller, B., Loeb, S., \& Kagan, S. (2004). Child Care in Poor Communities: Early Learning Effects of Type, Quality, and Stability. Child Development., 75 (1) 47 $-65$.

Caspary, G., Fuller, B., Gauthier, C., \& Kagan, S., (2002). Welfa- 
re Reform and Child Care Options for Low-Income Families. The Future of Children, 12 (1) 96-119.

COLCIENCIAS (2009). El desarrollo infantil en entornos urbanos pobres y contextos de interacción. Ref. 1106331-18930.

DeLoache, J., Miller, K. \& Pierroutsakos, S. (1998). Reasoning and Problem Solving. In: W. Damon, D. Khun \& R. Siegler (Eds). Handbook of Child Psychology. Cognition, Perception and language, Fifth edition, Vol. 2, 801-850. New York: Wiley \& Sons.

Gauvain, M \& Rogoff, B (1989). Collaborative Problem Solving and Children's Planning Skills. University State, University of Utah. Developmental Psychology, 25 (1). Pag. $139-15$.

Gauvain, M. (1995) Thinking in Niches: Sociocultural influences on Cognitive Development. Human Development. Vol 38, pp 25-45.

Gauvain, M. (2001). Cultural tools, social interaction and the development of thinking. Human Development, 44, $126-143$.

Gauvain, M. \& Perez, S. (2005). Parent-Child Participation in Planning Children's Activities Outside of School in European American and Latino Families. Child Development, March/April 2005, Volume 76, N. 2, pp. $371-383$

Gleason, M. \& Schauble, L. (2000). Parents' Assistance of Their Children's Scientific Reasoning. Cognition and Instruction, Vol. 17, No. 4. (1999), pp. 343-378.

Greenfield, P. \& Maynarda, A. (2003). Desarrollo cognoscitivo implícito en herramientas culturales y niños: lecciones del maya México. Desarrollo cognoscitivo, 18 489-510

Guevara, M. (2015). "Peer Interaction and Scientific Reasoning in Preschoolers. An Intra-individual Approach" In: Países Bajos, ed:University of Groningen

Guevara, M., van Dijk, M., \& van Geert, P. (2016). Microdevelopment of peer interactions and scientific reasoning in young children. Infancia y Aprendizaje, 39(4), 727-771.

Hojholt, C. (2005). El desarrollo Infantil a través de sus contextos sociales. Psicología y Ciencia Social. 7 (1 y 2). Universidad Nacional Autónoma de México, Distrito Federal, México. Pag. 22 - 40, ISSN 1405-5082.

Johnson, B. \& Onwuegbuzie, A. (2004). Mixed Methods Research: A Research Paradigm Whose Time Has Come. Educational Researcher, 33(7), 14-26.

Karmiloff, A. (1994). Más allá de la Modularidad. Madrid: Alianza Editores.

Lee, K. (2000). Childhood Cognitive Development: The Essential Readings. Malden: Blackwell.

Lippard, C., Riley, K \& Hughes-Belding, K., (2016). Observing toddlers' individual experiences in classrooms: initial use of the parenting interactions with children: checklist of observations linked to outcomes. Infant Mental Health Journal., 37(5), 549-559.

Orozco, M., Perinat, A. \& Sánchez, H. (2009). Cognitive Development and interaction Contexts. En A. C. Bastos \& E. Prabinovich (Eds.), Living in poverty: Developmental Poetics of Cultural Realities. (pp 287-308). Charlott, NC: Information Age Publishing.

Orozco, M., Sánchez, H \& Cerchiaro, E. (2012). Relación entre desarrollo cognitivo y contextos de interacción familiar de niños que viven en sectores urbanos pobres Univ. Psychol. V. 11 No. 2, p.p 427-440.
Piaget, J. \& Inhelder, B. (1976). Génesis de las estructuras lógicas elementales: clasificaciones y seriaciones. Buenos Aires: Editorial Guadalupe.

Perinat, A (2003). Psicología del Desarrollo: Un Enfoque Sistémico. Barcelona: Editorial

Puche, R. (2003). El niño que piensa y vuelve a pensar. Cali: Artes Gráficas del Valle Editores.

Puche, R. (2009) ¿Es la mente no lineal?" Cali: Programa Editorial Universidad Del Valle

Rogoff, B. (1993). Aprendices del pensamiento. El desarrollo cognitivo en el contexto social. Barcelona: Piadós.

Sánchez, H. \& Cuadros, Z. (2014). Perspectivas conceptuales para abordar estudios sobre relaciones afectivas tempranas. Encuentros, No. 1, 105-116.

Sánchez, H. (2017). Processos inferenciais e alteridade em elaborações do mito Madre Ñame da cultura Wounaan-nonam. Doctoral Thesis, Instituto de Psicologia, University of São Paulo, São Paulo. doi: 10.11606/T.47.2018.tde-22012018-103913.

Saxe, G., Guberman, S.R. \& Gwarhart M. (1987). Social processes in early number development. En: Monographs of the Society for Research in Child Development. $52(2)$.

Simão, L. M. (2010). Ensaios Dialógicos: compartilhamento e diferença nas relações eu -outro. Sao Pablo: Editora Hucitec.

Simão, L.M. \& Sanchez, H. (2017). "Cultural Practices as Scenario for Non-formal Education of Children in the Nonam Community of the Colombian Pacific Coast" In: Development Studies Discourse. Cultural Discourses Studies Series.

Valsiner, J. (2007). Culture in mind and societies- Fundations of cultural psychology. LA - London: SAGE.

Van Geert, P. (1998). A Dynamic Systems model of basic developmental mechanisms: Piaget, Vygotsky and beyond. In: Psychological Review, 105, Vol 5, No 4, 634-677 